\title{
Hybrid PSO Based-Variable Translation Wavelet Neural Network and Its Application To Hypoglycemia Detection System
}

\author{
Phyo Phyo San · Sai Ho Ling · Hung T. Nguyen
}

Received: date / Accepted: date

\begin{abstract}
To provide the detection of hypoglycemic episodes in Type 1 diabetes mellitus (T1DM), hypoglycemia detection system is developed by the use of variable translation wavelet neural network (VTWNN) in this paper. A wavelet neural network with variable translation parameter is selected as a suitable classifier because of its excellent characteristics in capturing nonstationary signal analysis and nonlinear function modeling. Due to the variable translation parameters, the network becomes an adaptive network and provides better classification performance. An improved hybrid PSO is used to train the parameters of VTWNN. Using the proposed classifier, a sensitivity of $81.40 \%$ and a specificity of $50.91 \%$ were achieved. The comparison results also show that the proposed detection system performs well in terms of good sensitivity and acceptable specificity.
\end{abstract}

Keywords Wavelet Neural Network · Particle Swarm Optimization · Diabetes and Hypoglycemia

\section{Introduction}

Intelligent technologies are essential for many biomedical engineering applications in order to perform different medical diagnosis purposes with various patients conditions. With the development of wavelet neural network, a combination of feedforward neural network and wavelet theory, a noninvasive hypoglycemia monitor is aimed to developed for Type 1 diabetes mellitus (T1DM) patients.

In the past few years, glucose meter has been the method choice for measurement of blood glucose concentration for

Phyo Phyo San · Sai Ho Ling · Hung T. Nguyen

Center for Health Technologies,

Faculty of Engineering and Information Technology,

University of Technology Sydney, Australia.

Tel.: +61-2-95149231

E-mail:PhyoPhyo.San@student.uts.edu.au the patients with T1DM. However, such kind of measurements can only provide isolated glucose values which do not reflect variations occurring throughout day and night. Due to discomfort and inconvenience of finger-stick methods, a non-invasive hypoglycemia monitoring system is tested and introduced.

A number of non-invasive hypoglycemia monitoring systems have been developed, for instance, Teledyne Sleep Sentry [1] [2] which is equipped with a sensor in order to measure fallen skin temperature and skin resistance. However, the disrupted sleep cycle occurred due to more false positive alarms. In multiparameter measurement system [3], electroencephalogram (EEG), pulse and skin impedance were used to monitor the status hypoglycemia, however the parameter, skin impedance is less correlation to status of hypoglycemia.

The Mini Med Medtronic CGMS [4] and Abortt Freestyle Navigator CGMS [5] are not recommended to use as alarms due to large mean absolute relative difference (MARD), $10 \%$ to $15 \%$. Although real-time continuous hypoglycemia monitoring systems are now available in the market [6][7], none of these are inefficient to use as alarms due to lack of sensitivity and low efficiency in detecting hypoglycemia.

With a focus on improving sensitivity and accuracy of hypoglycemia monitoring system, computational intelligence technologies have been applied to the development of hypoglycemia monitoring system such as evolved fuzzy reasoning model [10], multiple regression with fuzzy interference system [9], fuzzy neural network estimator [11], support vector machine [12], bayesian neural network [13] [14] have been developed by the use of physiological parameters of ECG signal. Though satisfactory sensitivity and specificity are obtained, achieving better sensitivity and specificity in detection of hypoglycemic episodes is still aiming and always finding out a better way by the use of advanced computational intelligent techniques. 
To achieve this, wavelet neural network [15] [16] has been selected as a suitable classifier due to its merit in capturing nonstationary signal analysis and nonlinear function modeling. It also has been proven that wavelet neural network (WNN) [17] [18] is a good classifier for nonstationary nature of ECG signal and provides faster convergence rate for approximation [19]

However, a typical WNN structure offers a fixed set of weight after training process and its fixed structure is unable to capture the characteristics of all input data. It is not be enough to learn the input data sets with fixed set of weight neural network if the data are separately distributed in a vast domain and/or the number of network parameters are too small. In this application, an improved variable translation wavelet neural network (VTWNN) is developed for modeling and design of non-invasive hypoglycemia detection system. In the proposed network, the translation parameters are variables depending on the network inputs and the network becomes more adaptive and provides better performance than conventional wavelet neural networks (WNNs).

Obtaining a set of optimal network parameters is one of the most important issues and gradient method [20] has been commonly used for training of the network parameters. However, it may only converge to a local optimum and is sensitive to values of initial parameters. The need of derivative information of optimized function become the main barrier and the learning method is only suitable for some specific network structure.

To overcome the drawbacks of trapping local optima, global search algorithms such as particle swarm optimization (PSO) [21], genetic algorithm [22] and simulated annealing [23] have been the method choices for the training of neural networks. They can also be used to train many different networks regardless of whether they are feed-forward, wavelet and any other type of network structures. In general, it saves a lot of time and effort in developing the training algorithms for different types of network. In this paper, we applied a hybrid particle swarm optimization with wavelet mutation (HPSOWM) [24] to train the VTWNN. In [24], which proven that the solution quality and solution stability are improved compared with other existing hybrid PSO methods.

The organization of this paper is as follows: in Section 2, variable translation wavelet neural network and their training procedures by the use of HPSOWM is introduced. To show the effectiveness of our proposed methods, the results of early detection of hypoglycemic episodes in T1DM are discussed in Section 3 and a conclusion is drawn in Section 4.

\section{METHODS}

An optimized variable translation wavelet neural network (VTWNN) with 4 inputs and 1 output system is developed for the detection of hypoglycaemic episodes in T1DM patients as shown in Fig. 1. The four psychological inputs of ECG signal are heart rate (HR), corrected QT interval (QTc), change of heart rate $(\Delta \mathrm{HR})$ and change of corrected QT interval in time $(\Delta \mathrm{QTc})$ while the output represents the status of hypoglycaemia $(h)(+1$ is hypoglycaemia and -1 is nonhypoglycaemia). The proposed VTWNN detection system is mainly used to find out the relationship between physiological parameters (HR, QTc, $\Delta \mathrm{HR}$ and $\Delta \mathrm{QTc})$ and the presence of hypoglycemia $(h)$.

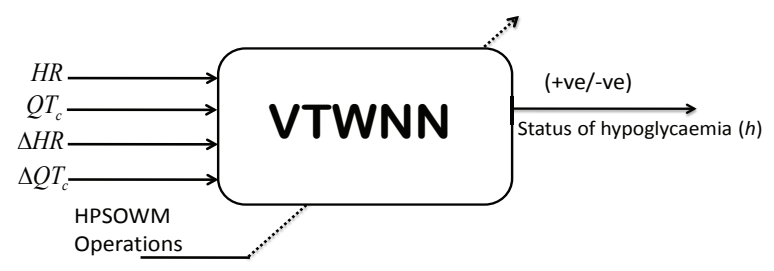

Fig. 1 VTWNN based hypoglycemia detection system

In order to detect the nocturnal hypoglycemia through analysis of ECG of Type 1 diabetes, the feature of ECG signal is extracted and classified according to their corresponding glucose levels. The typical ECG signal which is composed of a P Wave, a QRS complex and a T wave is presented in Fig. 2. The $\mathrm{P}$ wave represents atrial depolarization and the QRS represents ventricular depolarization while $\mathrm{T}$ wave reflects the phase of rapid repoplarization of the ventricles.

For patient with Type 1 diabetes, the possibility of hypoglycemia induced arrythmia is mainly effected by prolongation of QT interval, starting from the point of Q wave to at the end of $\mathrm{T}$ wave as shown in Fig. 2. The correlation of QT for heart rate is carried out by Bazett's formula $Q T_{c}=Q T / R R$. Not only $Q T_{c}$ interval prolongation has a significant impact on hypoglycemia, but an increase in heart rate (HR) also may influence the status of hypoglycemia [25][26].

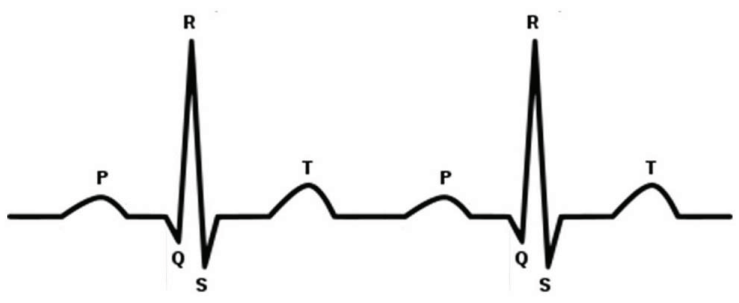

Fig. 2 ECG Signal 
The wavelet neural network is considered as a particular case of feedforward neural network and the neural network using wavelet basis function can provide faster convergence rates for approximation compared with conventional feedforward neural network. In addition, the wavelet has been applied in many research areas because of its excellent property in time-frequency localization of a given signal [27] [28]. By combining wavelet with neural network, wavelet neural network (WNN) has been developed in order to give better performance in function approximation and learning capabilities [29]. It can also be considered as a particular case of feedforward neural network (FFNN) apart from using multi-scaled wavelet activation function, $\psi_{a, b}(x)$ in the hidden layer.

By the use of wavelet as activation functions of the hidden layer, the dilation parameters, $a$ and translation parameter, $b$ of the wavelet is variable and definable with any real positive number. However, a conventional WNN is fail to capture the characteristics of separately distributed input data because it gives a fixed set of weight after training process. To overcome with this problem, a VTWNN with multiscale wavelet function is proposed in this paper.

Since the translation parameter, $b$ of proposed VTWNN is depending on the neural network inputs, the proposed VTWNN has ability to model the input-output function as the input-dependent network parameters. With the variable translation parameter, the proposed VTWNN gives faster learning ability with better generalization compared with other conventional neural networks [30]. It can be regarded as an adaptive neural network which has capability to handle different input patterns and gives a better performance. In this proposed system, the proposed VTWNN is trained by the use of HPSOWM.

\subsection{Variable Translation Wavelet Neural Network (VTWNN)}

The detail design and analysis of the variable translation wavelet neural network (VTWNN) will be discussed in this section. As presented in Fig. 3, the proposed VTWNN has a three-layer structure and its hidden layer input, $S_{j}$ is calculated as follows:

$S_{j}=\sum_{i=1}^{n_{\text {in }}} z_{i} v_{j i}, \quad j=1,2, \ldots, n_{h}$

where $z_{i}\left(i=1,2, \ldots, n_{i n}\right)$ are the inputs, and $v_{j i}$ denotes the weight between $i$ th input and $j$ th hidden nodes.

In order to control the magnitude and position of wavelet, multi-scaled wavelet function is used as the hidden node activation function. The hidden layer output of of $j$ th hidden neuron of proposed VTWNN is obtained in the following form:

$\psi_{j, b_{j}}=\frac{1}{\sqrt{j}} \psi\left(\frac{S_{j}-b_{j}}{j}\right)$

In this network, the Maxican Hat function is used as mother wavelet, $\psi(x)$, which is denoted as follows:

$\psi(x)=\left(1-x^{2}\right) \exp \left(-\frac{x^{2}}{2}\right)$

From Eq. (2) and Eq. (3), the hidden layer of VTWNN is obtained by:

$\psi_{j, b_{j}}=\frac{1}{\sqrt{j}}\left(1-\left(\frac{S_{j}-b_{j}}{j}\right)^{2}\right) \exp \left(-\frac{\left(\frac{S_{j}-b_{j}}{j}\right)^{2}}{2}\right)$

The translation parameter, $b_{j}$ is depending on the input $S_{j}$ and is governed by a nonlinear function $f^{j}(\cdot)$ as follows:

$b_{j}=f^{j}\left(S_{j}\right)=4 j\left(\frac{2}{1+e^{-K_{j} \times S_{j}}}-1\right)$

where $K_{j}$ is a tuned parameter that is used to control the shape of nonlinear function, $f^{j}(\cdot) . K_{j}$ The value of of $K_{j}$ is selected from interval [ 0.31 .5$]$. The value of $K$ should not be too small or too large. It behaves as a threshold function when $K \rightarrow \infty$, and it becomes a constant line when $k \rightarrow$ $-\infty$. The effect on parameter $K_{j}$ on the characteristics of nonlinear function $f^{j}(\cdot)$ in (5) is shown in Fig. 4.

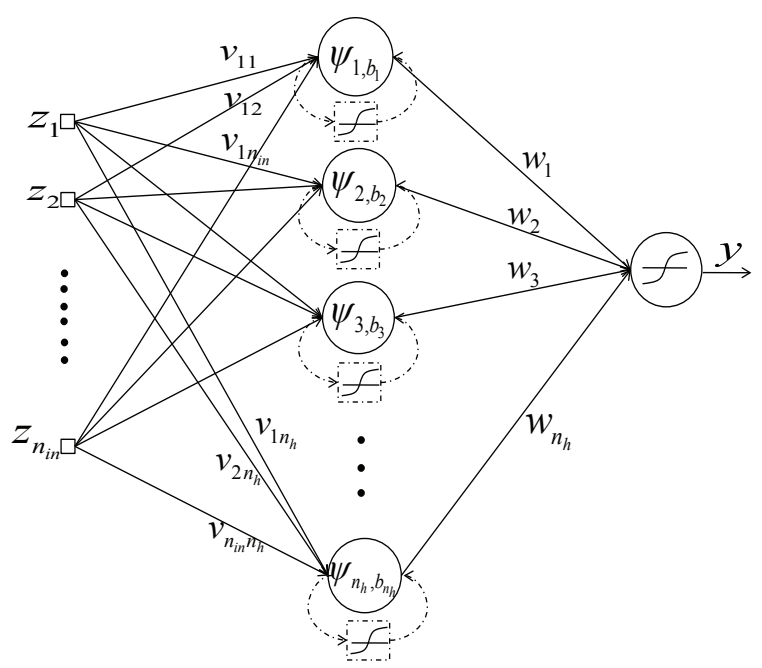

Fig. 3 Structure of Variable Translation Wavelet Neural Network

From Eq. (5) the value of translation parameters, $b_{j}$ is depending on the network related input $S_{j}$ and the nonlinear function parameter $K_{j}$. By doing so, the neural network is handling with variable translation parameter, $b_{j}$ with respect 
to neural network input parameter, $S_{j}$ in Eq.(1). Thus, the network becomes adaptive network and the output of proposed VTWNN is calculated by Eq. (1) - (5):

$y_{l}=\sum_{j=1}^{n_{h}} \psi_{j, b_{j}} w_{l j}$

where $w_{l j}, j=1,2, \ldots, n_{h}$ and $l=1,2, \ldots, n_{\text {out }}$ denotes the weight between the $j$ th hidden layer and $l$ th output layer. The network parameters of VTWNN are $v_{j i}, w_{l j}$ and $k_{j}$ will tuned by the used of HPSOWM optimization algorithm in Sec. 2.2.

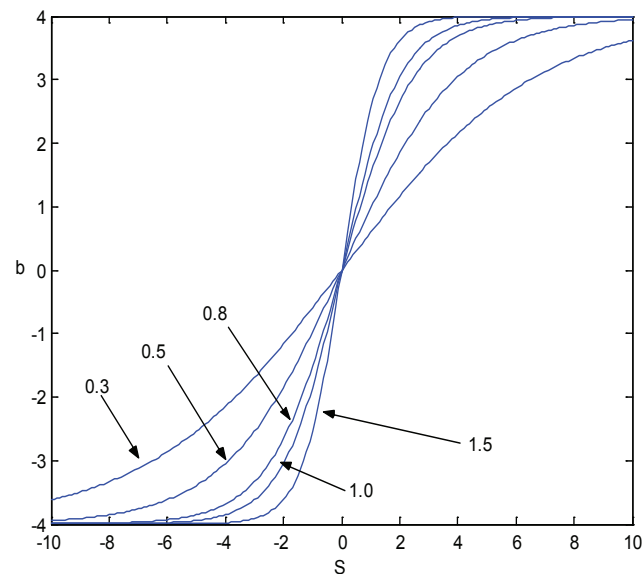

Fig. 4 Nonlinear function with different values of parameter $K$

\subsection{Hybrid Particle Swarm Optimization with Wavelet Mutation (HPSOWM)}

In HPSOWM, a swarm $X(t)$ is constituted with the number of particles. Each particle $\mathbf{x}^{p}(t) \in X(t)$ contains $\kappa$ elements $x_{j}^{p}(t)$ at the $t$-th iteration, where $p=1,2, \ldots, \theta$ and $j=$ $1,2, \ldots, \kappa ; \theta$ denotes the number of particles in the swarm and $\kappa$ is the dimension of a particle. First, the particles of the swarm are initialized and then evaluated by a defined fitness function.

The objective of HPSOWM is to minimize the fitness function (cost function) $f(X(t))$ of particles iteratively. The position $x_{j}^{p}(t)$ and velocity $v_{j}^{p}(t)$ used in HPSOWM are given as follows:

$$
\begin{aligned}
x_{j}^{p}(t) & =x_{j}^{p}(t-1)+v_{j}^{p}(t) \\
v_{j}^{p}(t) & =k \cdot\left(w \cdot v_{j}^{p}(t-1)+\varphi_{1} r_{1}\right) \cdot\left(\tilde{x}_{j}-x_{j}^{p}(t-1)\right) \\
& +\varphi_{2} r_{2}\left(\hat{x}_{j}-x_{j}^{p}(t-1)\right)
\end{aligned}
$$

where $\tilde{x}^{p}=\left[\tilde{x}_{1}^{p}, \tilde{x}_{2}^{p}, \ldots, \tilde{x}_{k}^{p}\right]$ and $\hat{\mathbf{x}}=\left[\begin{array}{ll}\hat{x_{1}} & \hat{x_{2}}, \ldots \hat{x_{\kappa}}\end{array}\right]$, $j=1,2, \ldots, \kappa$. The best previous position of a particle is recorded and represented as $\tilde{x}$; the position of best particle among all the particles is represented as $\hat{x} ; w$ is an inertia weight factor; $r_{1}$ and $r_{2}$ are acceleration constants which return a uniform random number in the range of $[0,1] ; w$ is inertia weight factor and $k$ is a constriction factor which detail derivation is discussed in [30].

Though PSO works well in the early stage, it leads to the problem of stagnation when a particle's current position is coincides with the global best position. To overcome this phenomenon, the mutation operation which starts with randomly chosen particle and excite them to move to different directions in the search area. For the hybrid PSO with mutation, a wavelet mutation (WM) that varies the mutating space based on wavelet theory is introduced because of its fine tune ability and solution stability.

To being with hybrid PSO with wavelet mutation (HPSOWM), a probability of mutation, $\mu_{m} \in\left[\begin{array}{ll}0 & 1\end{array}\right]$ is defined. For each particle element, a random number between 0 and 1 will be generated. If the generated number is less than or equal to $\mu_{m}$, the mutation will take place on that element. For instance, if $x^{p}(t)=\left[x_{1}^{p}(t), x_{1}^{p}(t), \ldots, x_{\kappa}^{p}(t)\right]$ is the selected $p^{t h}$ particle and the element of particle $x_{j}^{p}(t)$ is randomly selected for mutation and it's value are inside the particle element's boundaries, $\left[\rho_{\min }^{j}, \rho_{\max }^{j}\right]$.

The resulting particle, $\bar{x}^{p}(t)=\left[\bar{x}_{1}^{p}(t), \bar{x}_{1}^{p}(t), \ldots, \bar{x}_{\kappa}^{p}(t)\right]$ is governed by:

$\bar{x}_{j}^{p}(t)= \begin{cases}x_{j}^{p}(t)+\sigma \times\left(\rho_{\max }^{j}-x_{j}^{p}(t)\right) & , \sigma>0 \\ x_{j}^{p}(t)+\sigma \times\left(x_{j}^{p}(t)-\rho_{\min }^{j}\right) & , \sigma<0\end{cases}$

where $j \in 1,2, \ldots, \kappa$ and $\kappa$ denotes the dimension of particles. The value of $\sigma$ which is governed by Morlet wavelet function in the following form:

$\sigma=\psi_{a, 0}(\varphi)=\frac{1}{\sqrt{a}} \psi\left(\frac{\varphi}{a}\right)=\frac{1}{\sqrt{a}} e^{\frac{-\left(\frac{\varphi}{a}\right)^{2}}{2}} \cos \left(5\left(\frac{\varphi}{a}\right)\right)$

The amplitude of $\psi_{a, 0}(\varphi)$ will be scaled down as the dilation parameter $a$ increases. The mutation operation is used in order to enhance the searching performance. According to (9), a larger value of $|\sigma|$ gives a larger searching space for $x_{j}^{p}(t)$. When $|\sigma|$ is small, it gives a smaller searching space for fine-tuning. As over $99 \%$ of the total energy of mother wavelet function contained in the interval [-2.5 2.5], $\varphi$ can be randomly generated from $[-2.5 a 2.5 a]$.

The value of the dilation parameter $a$ is set to vary with the value of $\frac{t}{T}$ to meet the fine-tuning purpose, where $T$ is the total number of iteration and $t$ is the current number of iteration. In order to perform a local search when $t$ is large, the value of $a$ should increase as $\frac{t}{T}$ increases so as to reduce the significance of the mutation. Hence, a monotonic increasing function $a$ which is governing $a$ and $\frac{t}{T}$ is proposed in the following form:

$a=e^{-\ln (g) \times\left(1-\frac{t}{T}\right)^{\zeta w m}+\ln (g)}$

where $\zeta_{w m}$ is the shape parameter of the monotonic increasing function, $g$ is the upper limit of the parameter $a$. 
After the operation of wavelet mutation, a new swarm is generated and the same process will be repeated. Such an iterative process will be terminated if the pre-defined number of iterations is met.

The performance of HPSOWM is governed by the right choices of the control parameters such as Swarm size $(\theta)$, the probability of mutation $\left(\mu_{m}\right)$, the shape parameter $\left(\zeta_{w m}\right)$ and parameter $g$ of wavelet mutation. For swarm size $(\theta)$, increasing it's size will increase the diversity of the search space, and reduce the probability that HPSOWM prematurely converges to a local optimum. However, it also increases the time required for the population to converge to the optimal region in the search space.

The probability of mutation $\left(\mu_{m}\right)$ is largely depends on the desired number of element of particles that undergo the mutation operation. The value of $\mu_{m}$ is set from 0.1 to 1 based on the number of element of particles (dimension of particles). However, increasing the probability of mutation $\left(\mu_{m}=1\right)$ tends to transform the search into a random search and all element of particles will mutate. This probability gives us an expected number $\left(\mu_{m} \times \theta \times \kappa\right)$ of element of particles that undergo the mutation operation.

The dilation parameter $a$ is governed by the monotonic increasing function which is controlled by shape parameter $\zeta_{w m}$ and parameter $g$. Changing the parameter $\zeta_{w m}$ will change the characteristics of the monotonic increasing function of the wavelet mutation. When $\zeta_{w m}$ becomes larger, the decreasing speed of the step size $(\sigma)$ of mutation becomes faster. Similarity, a larger value of $g$ implies that the maximum value of dilation parameter $a$ and it is leading to smaller searching space since $\sigma$ is smaller. To control the monotonic increasing function, fix one parameters and tune another parameters is the best method for good setting.

\subsection{Fitness Function and Training}

The objective of proposed optimized VTWNN is to detect the hypoglycemic episodes accurately based on the psychological parameters of ECG singal, HR, QTc, $\Delta \mathrm{HR}$ and $\Delta \mathrm{QTc}$. To measure the performance of biomedical classification test, the sensitivity and specificity [31] are introduced as follows:

$$
\begin{aligned}
& \text { sensitivity }(\xi)=\frac{\text { True positives }}{\text { True positives }+ \text { False negatives }} \\
& \text { specifity }(\eta)=\frac{\text { True negatives }}{\text { True negatives }+ \text { False positives }}
\end{aligned}
$$

The sensitivity measures the proportion of number of true positives which implies the sick people correctly diagnosed as sick and number of false negatives which implies the sick people wrongly diagnosed as healthy, the specificity measures the proportion of true negatives which are correctly classified healthy people as healthy and the number of false positives which implied healthy people wrongly diagnosed as sick.

The objective is to maximize the fitness function of Eq. (11) which equivalent to maximize the sensitivity and the specificity. To meet the objective of the system the fitness function $f(\xi, \eta)$ is defined by using sensitivity, $\xi$ and specificity, $\eta$ as follow:

$$
f(\xi, \eta)=\xi+\frac{\eta_{\max }-\eta}{\eta_{\max }}
$$

where $\eta_{\max }$ is a upper limit of the specificity. In Eq. (11), the specificity is limited by a maximum value $\eta_{\max }$. In Eq. (11), the parameter $\eta_{\max }$ is used to fix the region of specificity in order to find the optimal sensitivity in this region. In particularly, the $\eta_{\max }$ can set from 0 to 1 and different sensitivity with different specificity value can be determined. The determination of optimal sensitivity with different specificity, $\eta_{\max }$ are examined in the following Section. 3.

The optimal parameters $v_{j i}, w_{l j}$ and $k_{j}$ is obtained through the training process with HPSOWM after maximizing the proposed fitness function in (11). In this application, the number of proposed VTWNN is mainly depend on the number of hidden neurons $n_{h}$ since the number of parameters for $v_{j i}$ is equal to $n_{i n} \times n_{h}$; the number of parameters for $w_{l j}$ and $K_{j}$ are equal to $n_{h}$. Thus, the total number of parameters is $n_{h}\left(n_{i n}+1+1\right)$. For a given set of particle $\mathbf{x}^{p}(t)$, HPSOWM evaluates the fitness value of the each particle at each iteration and searches for the optimum network parameters.

To find the optimal parameters of VTWNN, the parameters of the HPSOWM are selected as: swarm size $\theta=50$, constant value $c_{1}$ and $c_{2}=2.05$, maximum velocity $v_{\max }=$ 0.2 , probability of mutation $\mu_{m}=0.7$, the shape parameter of wavelet mutation $\zeta_{w m}=2$, the constant value $g$ of wavelet mutation $=10000$ and the number of iteration $T=2000$. The parameters $\theta, c_{1}, c_{2}, \zeta_{w m}$, and $g$ are recommended by [24]. $\mu_{m}$ and $T$ are selected by trial and error through experiments.

\section{RESULT AND DISCUSSION}

To study the natural occurrence of nocturnal hypoglycemia, 15 children with T1DM is monitored for the 10-hour overnight at the Princess Margaret Hospital for Children in Perth, Western Australia, Australia. The required physiological parameters are measured by the use of non-invasive monitoring system [32] while the actual blood glucose level (BGL) are collected using Yellow Spring Instruments to use as a reference. All the data are collected at the same time and the duration for each patients varies from 400 minutes to 480 minutes. 
The significant change of 15 T1DM children responses can be distinctly seen during they hypoglycemia phase against non-hypoglycemia phase in the actual blood glucose profile for 15 T1DM children [9]. The presence of hypoglycemia are estimated at sampling period $k_{s}$ and the previous data at sampling period $k_{s}-1$. In general, the sampling period takes about 5 to 10 minutes and approximately 35-40 data points are used for each patient. Since normalization is carried out, patient-to-patient variability is reduced and group comparison is enabled by dividing the patient's heart rate (HR), corrected QT interval (QTc) by their corresponding value at time zero.

The overall data set consist of both hypoglycemia data part and non-hypoglycemia data part and it is organized into a training set (5 patients with 184 data points), a validation set (5 patients with 192 data points) and a testing set (5 patients with 153 data points) which are randomly selected. To tackle the problem of T1DM, the hypoglycemia episodes $(\mathrm{BGL} \leq 3.3 \mathrm{mmol} / \mathrm{l})$ is detected by the use of HPSOWM based optimized variable translation wavelet neural network (VTWNN).

The main parameters used for the detection of hypoglycemia are the heart rate (HR), corrected QT (QTc) interval, change of heart rate $(\Delta \mathrm{HR})$ and change of corrected QT interval $(\Delta \mathrm{QTc})$ because of their higher correlation with hypoglycemic episodes [33]. Regarding with the correlation between hypoglycemia and cardiac dysrhythmia, a number of studies have been demonstrated that hypoglycemia mainly alter the ventricular repolarization and prolong the duration of QT interval of ECG signal in Fig. 2. The relationship between an increased in normalized heart rates $(1.033 \pm 0.242$ vs. $1.082 \pm<0.06)$, correct QT interval $(1.031 \pm 0.086$ vs. $1.060 \pm 0.084<0.001)$ and their associated hypoglycemia status has been studied [14].

In this application, three comparison approaches such as variable translation wavelet neural network (VTWNN), wavelet neural network (WNN) and feedforwrad neural network (FWNN) are compared and analyzed. All approaches are trained with HPSOWM. After the training process, the performance of proposed detection system is analyzed by means of ROC curve in Fig. 5 in which the sensitivity (true positive rate) and the 1 -specificity (false positive rate) are relatively plotted. In ROC curve analysis, the accuracy is measured by the area under the ROC curve and the wider ROC curve area is defined as the better accuracy.

As can be seen in Fig. 5, the corresponding ROC curve areas for VTWNN, WNN and FWNN are compared and analyzed. The study compared methods in VTWNN, WNN and FWNN found that the proposed VTWNN perform more accurately in detection of hypoglycemia (area under the curve $=0.7082$ ) compared to WNN and FWNN (area under the curve $=0.6753$ and 0.6695 ).
In this studies, the gamma analysis $(\gamma=\theta \xi+(1-\theta) \eta$, $\theta \in[0.1,1])$ is defined to evaluate the performance of proposed VTWNN detection system. The value of $\theta$ is set to 0.6 since the minimum requirement of hypoglycemia detection system is $60 \%$ of sensitivity and $40 \%$ of specificity. In clinical study, the sensitivity of detection system most important than the specificity because it mainly represents the performance of classifier for patients with hypoglycemia.

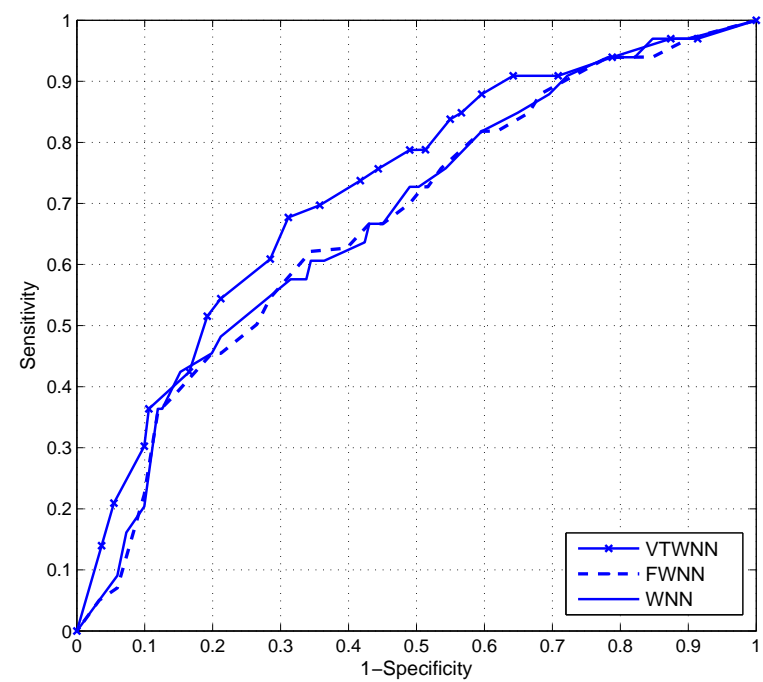

Fig. 5 ROC curve analysis

By the use of ROC curve in Fig. 5, the mean (average) sensitivity and specificity of training, validation and testing are compared and analyzed by fixing the cut-off point at 0.6 $\left(1-\eta_{\max }=60 \%\right)$ which is equivalent to maximum specificity, $\eta_{\max }=40 \%$. The mean results in term of the sensitivity and specificity are tabulated in Table 1 . All results are averaging over 20 runs.

In Table 1, the proposed optimized VTWNN is found to be satisfactory by giving mean (average) testing sensitivity and specificity value of $77.41 \%$ and $47.42 \%$ while the other comparison methods WNN amd FWNN are giving (71.39\% and $44.37 \%$ ) and ( $68.84 \%$ and $48.34 \%$ ). In terms of $\gamma$ analysis, the proposed optimized VTWNN gives better performance $(\gamma=65.41 \%)$ while the other comparison methods: WNN and FWNN have $\gamma$ value of $60.58 \%, 60.64 \%$ and $60.03 \%$. 
Table 1 Mean Vale of Training Validation and Testing Results: Set maximum specificity, $\eta_{\max }=40 \%$

\begin{tabular}{|c|c|c|c|c|c|}
\hline$\eta_{\max }$ & & & VTWNN & WNN & FWNN \\
\hline \multirow{9}{*}{$40 \%$} & \multirow[t]{3}{*}{ Training (\%) } & $\operatorname{Sen}(\xi)$ & 88.40 & 84.12 & 83.64 \\
\hline & & $\operatorname{Spec}(\eta)$ & 40.80 & 40.63 & 40.50 \\
\hline & & $\gamma$ & 69.36 & 66.72 & 66.38 \\
\hline & \multirow[t]{3}{*}{ Validation (\%) } & $\operatorname{Sen}(\xi)$ & 82.50 & 80.44 & 79.07 \\
\hline & & $\operatorname{spec}(\eta)$ & 41.25 & 40.94 & 41.38 \\
\hline & & $\gamma$ & 66.00 & 64.64 & 63.99 \\
\hline & \multirow[t]{3}{*}{ Testing (\%) } & $\operatorname{Sen}(\xi)$ & 77.41 & 71.39 & 68.84 \\
\hline & & $\operatorname{Spec}(\eta)$ & 47.42 & 44.37 & 48.34 \\
\hline & & $\gamma$ & 65.41 & 60.58 & 60.64 \\
\hline
\end{tabular}

In order to prove that the optimized VTWNN gives better classification performance, the analysis is continuously carried out at the different cut-off points at $\eta_{\max }=40 \%, 60 \%$ and $80 \%$. As presented in Table 2, the proposed VTWNN achieves best testing sensitivity and acceptable specificity of $81.40 \%$ and $50.91 \%$ whereas WNN and FWNN give best testing sensitivity and specificity of $(74.42 \%$ and $48.18 \%)$ and $(69.77 \%$ and $49.09 \%)$. Similarity, at the defined cutoff points $\eta_{\max }=60 \%$ and $80 \%$, the proposed VTWNN gives better sensitivity and specificity compared with other classifiers.

From Table. 3, it can be seen that the optimized VTWNN achieves better testing sensitivity and specificity compared with other neural network classifiers (WNN, FWNN), evolved fuzzy interference system (FIS) and a genetic algorithm (GA) based multiple regression with fuzzy interference system (MRFIS).

Table 2 Best Testing Result for Hypoglycemia Detection with Different Approaches: Set maximum specificity, $\eta_{\max }=40 \%, 60 \%$, and $80 \%$

\begin{tabular}{cllll}
\hline$\eta_{\max }$ & & VTWNN & WNN & FWNN \\
\hline \hline \multirow{2}{*}{$40 \%$} & Sen $(\xi)$ & $\mathbf{8 1 . 4 0}$ & 74.42 & 69.77 \\
\cline { 2 - 5 } & Spec $(\eta)$ & $\mathbf{5 0 . 9 1}$ & 48.18 & 49.09 \\
\cline { 2 - 5 } & $\gamma$ & $\mathbf{6 9 . 2 0}$ & 63.92 & 61.50 \\
\hline \hline \multirow{2}{*}{$60 \%$} & Sen $(\xi)$ & $\mathbf{5 3 . 4 9}$ & 48.84 & 44.88 \\
\cline { 2 - 5 } & Spec $(\eta)$ & $\mathbf{6 8 . 1 8}$ & 72.73 & 70.00 \\
\cline { 2 - 5 } & $\gamma$ & $\mathbf{5 9 . 3 4}$ & 58.40 & 54.93 \\
\hline \hline \multirow{3}{*}{$80 \%$} & Sen $(\xi)$ & $\mathbf{3 7 . 9 1}$ & 23.16 & 20.93 \\
\cline { 2 - 5 } & Spec $(\eta)$ & $\mathbf{8 4 . 5 5}$ & 90.17 & 93.28 \\
\cline { 2 - 5 } & $\gamma$ & $\mathbf{5 6 . 5 7}$ & 49.96 & 49.87 \\
\hline
\end{tabular}

Table 3 Best Testing Result for Hypoglycemia Detection with Neural and Fuzzy Classifiers

\begin{tabular}{llll}
\hline Methods & Sensitivity & Specificity & $\gamma$ \\
\hline \hline VTWNN & $\mathbf{8 1 . 4 0}$ & $\mathbf{5 0 . 9 1}$ & $\mathbf{6 9 . 2 0}$ \\
\hline MR-FIS [9] & 75.00 & 50.00 & 65.00 \\
\hline WNN & 74.42 & 48.18 & 63.92 \\
\hline FRM [8] & 70.45 & 55.14 & 64.32 \\
\hline FWNN & 69.77 & 49.09 & 61.50 \\
\hline MR & 65.12 & 57.27 & 61.98 \\
\hline
\end{tabular}

As can be seen in Table. 3, the optimized VTWNN gives the best testing sensitivity of $81.40 \%$ while the specificity is kept around $50 \%$. Based on the common criteria of clinical classification (sensitivity $\geq 0.7$ and specificity $\geq 0.5$ ), from Table $1-3$, it can be distinctly seen that the optimized VTWNN is effective and best suited for detection of hypoglycemic episodes. In summary, the effectiveness of optimized VTWNN detection system can be distinctly seen in Table 1 - 3 with the better classification performance $(81.40$ $\%$ of Sensitivity and $50.91 \%$ of acceptable testing specificity).

\section{CONCLUSIONS}

This paper presents an application of hybrid particle swarm optimization based variable translation wavelet neural network (VTWNN) for the detection of hypoglycemic episodes in T1DM patients. By the use of real time physiological inputs, the hypoglycemia episodes in T1DM children can be efficiently detected the status of hypoglycemia non-invasively and continuously.

Due to variable translation parameters in the network, the proposed VTWNN becomes adaptive network and has ability to improve network learning ability. The results indicate that the optimized VTWNN detection system gives better testing sensitivity of $81.40 \%$ and specificity of $50.91 \%$ compared with other classifiers, WNN, FWNN, FIS, MRFIS and MR. Product design and other industrial applications will be further examined in order to validate the effectiveness of the proposed VTWNN.

\section{References}

1. K. A. Hansen and S. C. Duck, "Teledyne sleep sentry: evaluation in pediatric patients for detection of nocturnal hypoglycemia", Diabetes Care, vol.6, no.6, pp. 597-600, 1983.

2. J. Castano, Y. Z. Wang and D. Chuang, "Blood glucose dependence of visual flicker threshold", Diabetes Technology and Therapeutics, vol.2, pp. 31-43, 2000. 
3. G. Heger, K. Howorka and H. Thoma, "Monitoring set-up for selection of parameters for detection of hypoglycaemia in diabetic patients", Medical and Biological Engineering and Computing, vol.34, no.1, pp. 69-75, 1996.

4. F. F. R.Maia and L. R.Araujo, "Efficacy of continuous glucose monitoring system (CGMS) to detect postprandial hyperglycemia and unrecognized hypoglycemia in type 1 diabetic patients", Diabetes Research and Clinical Practice, vol.75, pp. 30-34, 2007.

5. R. L. Weinstein, "Accuracy of the freestyle navigator CGMS: Comparison with frequent laboratory measurements", Diabetes Care, vol.30, pp. 1125-1130, 2007.

6. Directnet Study Group, "Evaluation of factors affecting CGMS calibration", Diabetes Technology and Therapeutics, vol.8, pp. 318325, 2006.

7. C. E. F. Amaral and B. Wolf, "Current development in non-invasive glucose monitoring", Medical Engineering and Physics, vol.30, no. 5, pp. 541-549, 2008.

8. S. H. Ling, N. Nuryani and H. T. Nguyen, "Evolved fuzzy reasoning model for hypoglycaemic detection", Proceeding of the 32th Annual International Conference of the IEEE Engineering in Medicine and Biology Society, pp. 4662-4665, 2010.

9. S. H. Ling and H. T. Nguyen, "Genetic-Algorithm-Based Multiple Regression With Fuzzy Inference System for Detection of Nocturnal Hypoglycemic Episodes", IEEE Transactions on Information Technology in Biomedicine, vol.15, no.2, pp. 308-315, 2011.

10. S. H. Ling and H. T. Nguyen, "Natural occurrence of nocturnal hypoglycemia detection using hybrid particle swarm optimized fuzzy reasoning model", Artificial Intelligence in Medicine, vol. 55, no. 3, pp. 177-184, 2012

11. N. Ghevondian, H. T. Nguyen and S. Colagiuri, "A novel fuzzy neural network estimator for predicting hypoglycaemia in insulininduced subjects", Proceeding of the 23rd Annual International Conference of the IEEE Engineering in Medicine and Biology Society, pp. 1657-1660, 2001.

12. N. Nuryani, S. H. Ling and H. T. Nguyen, "Electrocardiographic signals and swarm-based support vector machine for hypoglycemia detection”, Annals of Biomedical Engineering, vol.40, no.4, pp. 934945, 2011.

13. H. T. Nguyen, N. Ghevondian, S. T. Nguyen and T. W. Jones, "Detection of hypoglycemic episodes in children with type 1 diabetes using an optimal Bayesian neural network algorithm", Proceedings of the International Conference of IEEE Engineering in Medicine and Biology Society, pp. 3140-3143, 2007.

14. H. T. Nguyen, N. Ghevondian and T. W. Jones, "Detection of nocturnal hypoglycemic episodes (natural occurrence) in children with Type 1 diabetes using an optimal Bayesian neural network algorithm", Proceeding of the 30th Annual International Conference of the IEEE Engineering in Medicine and Biology Society, pp. 13111314, 2008.

15. B. Biswal, P. K. Dash, B. K. Panigrahi and J. B. V. Reddy, "Power signal classification using dynamic wavelet network", Applied Soft Computing, vol. 9, no. 1, pp. $118-125,2009$.

16. P. K. Dash, B. Biswal and B. K. Panigrahi, "A new approach to time-frequency analysis and pattern recognition of non-stationary power signal disturbances", International Journal of Engineering Intelligent System, vol. 14, no. 1, pp. 3-14, 2006.

17. J. Yao, Q. Gan, D. X. Zhang, and J. Li, "Pruning algorithm in wavelet neural network for ECG signal classification", Proceedings of IEEE Engineering in Medicine and Biology Society , pp. 1482$1485,1998$.

18. I. Daubechies, "The wavelet transform, timefrequency localization and signal analysis", IEEE Transactions on Information Theory, vol. 36, no. 5, pp. 961-1005, 1990.

19. Q. Zhang and A. Benveniste, "Wavelet networks", IEEE Transactions on Neural Networks, vol. 3, no. 6, pp. 889 -898, 1992.

20. M. F. Moller, "A scaled conjugate gradient algorithm for fast supervised learning", Neural Network, vol.6, no. 4, pp. 525-533, 1993.
21. J. Kennedy and R. Eberhart, "Particle swarm optimization", Proceedings of IEEE International Conference on Neural Networks, pp. 1942-1948, 1995

22. K. F. Leung and F. H. F. Leung and H. K. Lam and S.H. Ling, "Application of a modified neural fuzzy network and an improved genetic algorithm to speech recognition",Neural Computing and Applications, vol. 16, no. 4, pp. 419-431, 2007.

23. K. Y. Chan and C. K. Kwong and X. G. Luo," Improved orthogonal array based simulated annealing for design optimization",Expert Systems with Application, vol. 36, no. 4, pp. 7379-7389, 2009.

24. S. H. Ling, H. C. C. Iu, K. Y. Chan, H. K. Lam, C. W. Yeung, and F. H. F Leung, "Hybrid particle swarm optimization with wavelet mutation and its industrial applications", IEEE Transactions on Systems, Man, and Cybernetics. Part B, vol.38, no.3, pp. 743-763, 2008.

25. N. P. , M. E. Ford-Adams and K. K. Ong, "Prolonged cardiac repolarisation during spontaneous nocturnal hypoglycaemia in children and adolescents with type 1 diabetes", Diabetologia, vol.47, pp. 1940-1947,2004.

26. S. P. Lee, L. Yeoh and N. D. Harris, "Influence of autonomic neuropathy on QTc interval lengthening during hypoglycemia in type 1 diabetes", Diabetes, vol.53, pp. 1535-1542,2004.

27. M. Zekri and S. Sadri, "Adaptive fuzzy wavelet network control design for nonlinear systems", Fuzzy Sets Systems, vol. 20, pp. 2668 $-2695,2008$.

28. S. G. Mallat, "A theory for multiresolution signal decomposition: The wavelet representation", IEEE Transactions on Pattern Analysis and Machine Intelligence, vol. 11, no. 7, pp. 674-693, 1989.

29. S. A. Billings and H. L. Wei, "A new class of wavelet networks for nonlinear system identification", IEEE Transactions Neural Networks, vol. 16, no. 4, pp. 862-874, 2005.

30. S. H. Ling, F. H. F. Leung and K. Y. Chan, "Improved Hybrid Particle Swarm Optimized Wavelet Neural Network for Modeling the Development of Fluid Dispensing for Electronic Packaging", IEEE Transactions on Industrial Electronics, vol. 55, no. 9, pp. 3447-3460, 2008.

31. D. G. Altman and J. M. Bland, "Statistics Notes: Diagnostic tests 1: sensitivity and specificity”, Clinical chemistry, vol.308, pp. 1552$1552,1994$.

32. H. T. Nguyen and N. Ghevondian and T. W. Jones, "NeuralNetwork Detection of Hypoglycemic Episodes in Children with Type 1 Diabetes using Physiological Parameters", 28th Annual International Conference of the IEEE Engineering in Medicine and Biology Society, pp. 6053-6056, 2006.

33. H. T. Nguyen and N. Ghevondian and T. W. Jones, "Real time detection of nocturnal hypoglycemic episodes using a novel noninvasive hypoglycemia monitor", 31st Annual International Conference of the IEEE Engineering in Medicine and Biology Society, pp. 3822-3825, 2008. 